\title{
ESPACIOS SUBORDINANTES Y SUBORDINADOS EN EL PROYECTO CONTEMPORANEO
}

\author{
Fernando Diez \\ Doctor Arquitecto Catedrático UB Buenos Aires. Director Summat \\ ASTRAGALO, 20 (2015) \\ Attribution-NonCommercial-ShareAlike - CC BY-NC-SA \\ Artículo, ISSN 2469-0503 \\ https://dx.doi.org/10.12795/astragalo.2015.i20.10
}

\section{RELACIÓN ENTRE DIBUJO Y PROYECTUALIDAD.}

Por su carácter plano, el dibujo es una reducción arbitraria de las cualidades morfológicas tridimensionales de lo representado a su posible graficación bidimensional. El convencionalismo en que se desenvuelve esta operación la transforma en automática, en un dato culturalmente asimilado. Se sistematiza en la enseñanza superior y su arbitrariedad se internaliza mentalmente. Aunque en cada disciplina hay una representación plana aceptada estas son arbitrarias en la decisión de qué es incluido y qué es excluido del dibujo. En especial en los dibujos producidos por los arquitectos para presentar sus proyectos a otros arquitectos, emerge una representación que se aparta de la convención para subrayar determinados aspectos, sirviendo de fundamento legitimante del proyecto. Valen como ejemplo los dibujos y fotomontajes de Le Corbusier realizados para ilustrar el Plan Voisin, tan distintos de los realizados por Colin Rowe sobre el mismo proyecto. Los primeros, destacando la oscuridad del espacio del Paris tradicional y minimizando la intensidad de la sombra de los rascacielos, los segundos ateniéndose a la oposición sólido vacío, poniendo de relieve la cualificación espacial del espacio urbano tradicional.

\section{[ver figura 1, página siguiente]}

En el dibujo de arquitectura la línea separa zonas compuestas por distinta materia. El muro de piedra del aire que contiene una habitación o rodea la casa. En algunos casos, esa diferencia se hace más explícita al llenarse completamente con el color de la línea, generalmente negra, la materia sólida del muro, diferenciándola más 

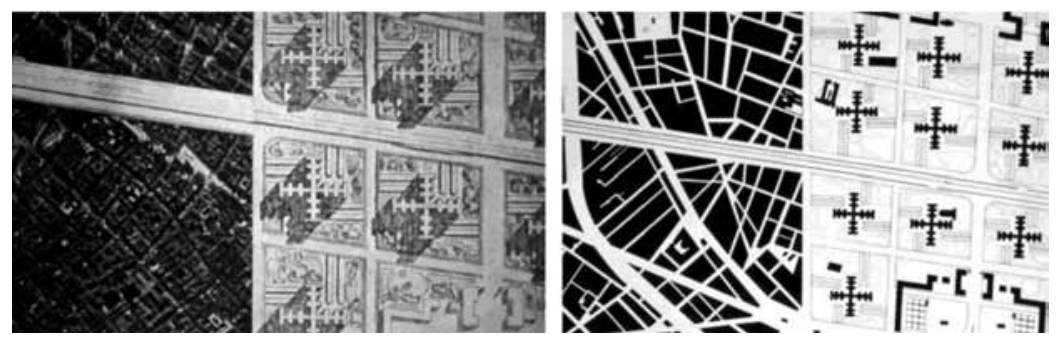

F1: Plan Voisin, según Le Corbusier y según la representación de Colin Rowe interiores visibles, y el espacio arquitectónico cualificado. Una sofisticada organización espacial, basada en la percepción de una figura espacial y un fondo constituido completamente de la materia gaseosa del aire, blanco. Sólido y gaseoso se equiparan a lleno y vacío, contenedor y contenido. En la tratadística clásica, el sólido corresponde a los "elementos de arquitectura” y el vacío a los elementos de composición ${ }^{1}$.

En la arquitectura beaux-arts, el sólido de los muros se denominaba poché y se representaba en color rosado. Este permitía configurar las habitaciones del modo deseado absorbiendo las diferencias formales entre ellas, una suerte de relleno, subsidiario de la forma de los espacios principales. La forma del poché es a la vez un agente y un sobrante, puede ser visto como un productor de forma y como un residuo de otras formas privilegiadas por el proyectista. Una forma subordinada a la voluntad de crear otra. El vacío es una forma voluntaria y el poché es su sobrante, confinado, en principio, al espesor variable de los muros. Pero también pueden serlo espacios huecos ocupados por armarios o pasos, incluso habitaciones secundarias o de menor tamaño. Aparece así un principio de organización en que la forma de los espacios principales es conseguida con la ayuda de la forma de muros y espacios menores. Ya no se trata de una oposición literal entre sólido y vacío, sino de una oposición conceptual entre aquello percibido como sólido, detrás de los paramentos por lo que desde determinada posición no es visible. Colin Rowe identifica esta correlación con el concepto de gestalt, donde la forma, la figura que ocupa la atención visual, aparece como oposición al fondo ${ }^{2}$. Queda así planteada una correlación entre las nociones de forma subordinada-sólido-fondo, opuesta a la de forma subordinante-espacio-figura.

Esta correlación puede ser intencionadamente sugerida por el dibujo de planta según la particular forma de representación. En la representación Beaux-arts, el color rosado hace explícita la cualidad subordinada de la forma. En los dibujos de las obras contemporáneas aquí consideradas de Aires Mateus, Herzog \& deMeuron y OMA, los autores producen una representación que hace explícitos los espacios subordinados y por lo tanto, también los espacios subordinantes. Al hacerlo, los arquitectos muestran, simultáneamente, la percepción esperada tanto como el procedimiento de proyecto utilizado. En estos casos, la tradicional correspondencia entre negro y sólido, en oposición a la correspondencia entre blanco y vacío, es alterada en una parte del dibujo, invirtiéndose. E invirtiendo también el valor de la línea, que pasa de su negro convencional, al blanco. Esta desviación del canon de la convención revela un procedimiento de organización del pro- 
yecto contemporáneo que recupera un interés en el espacio ${ }^{3}$, haciendo explícito un modo proyectual que vincula tres tradiciones de diseño: la clásica, la moderna y la contemporánea.

\section{ADICIÓN Y SUSTRACCIÓN}

La noción de que el proyecto de arquitectura es el resultado de una sumatoria de partes preexistentes es la natural consecuencia de imaginarlo como una sumatoria de habitaciones. Inversamente, la subdivisión de una gran habitación produce habitaciones menores. El perfeccionamiento de estos principios fue inherente a la arquitectura clásica y con Durand $^{4}$, la noción de composición se hace dominante. Noción que nunca abandonó del todo la arquitectura moderna. Le Corbusier dejó en sus obras completas un documento revelador que aparece a página completa. Las 4 composiciones, así explícitamente llamadas, ilustran cuatro modos de componer. Se trata de un menú de procedimientos compositivos: el primero, ilustrado con la Maison Roche, es visiblemente el resultado de la adición de formas contrastantes. El segundo, ilustrado con la Maison á Garches, muestra un paralelogramo que debe ser dividido interiormente para crear las habitaciones. El tercero, ejemplificado por la Maison á Stuttgart, sigue el principio de la Maison Dominó, mostrando la independencia entre columnas y muros. El cuarto, ejemplificado con la Ville Saboye, muestra un proceso sustractivo, donde el perímetro rectangular de la planta alta (casi un cuadrado) es de superficie mayor a la necesaria para el programa cubierto. El patio irregular es el resultado de una suerte de sustracción espacial producida por la ubicación de las habitaciones. Muy generoso, anota Le
Corbusier, subrayando que afirma al exterior una voluntad arquitectónica y satisface al interior las necesidades funcionales. Los dos ejemplos extremos representen adición (la Roche) y sustracción (Saboye). En ambos, esas operaciones son estrictamente mentales, es decir, que se verifican en el plano del proyecto, pero también son perceptibles a simple vista ${ }^{5}$.

1
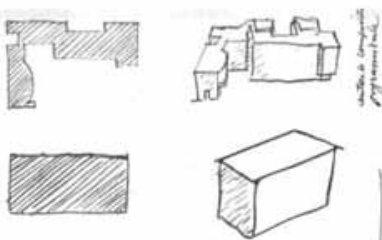

3
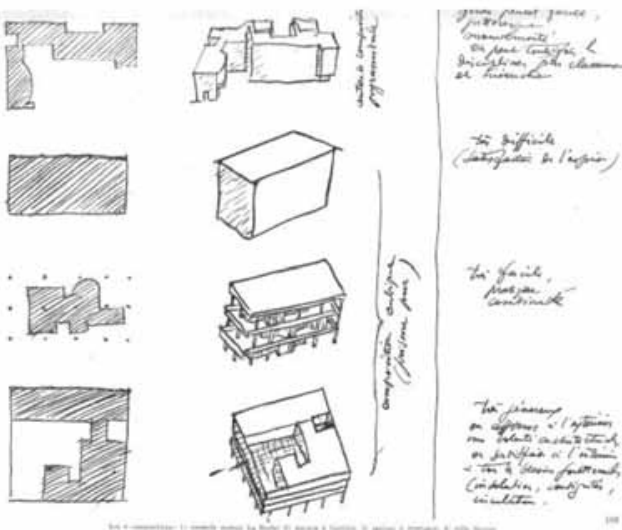

F2: las "4 composiciones" de Le Corbusier

\section{FORMAS SUBORDINANTES Y SUBORDINADAS}

Colin Rowe y Fred Koetter ${ }^{6}$ analizan la persistencia histórica de ciertos procedimientos de proyecto centrados en la oposición formal entre fondo y figura, tanto como en la capacidad organizativa de una forma dominante desde el punto de vista perceptivo, conceptos que se alimentaban de la teoría gestalt. En la comparación más elocuente proponen ver a la Galleria degli Uffizi en Florencia (Vasari, siglo XVI) como un espacio cuyo vacío es similar en forma y dimensiones al sólido objeto que constituye la Unité d'Habitation de Marsella (Le Corbusier, 
1946). Ambas correlaciones, fondo y figura, sólido y vacío, se encuentran indisolublemente ligadas. En el Hotel de Beauvais y en la planta alta de la Ville Saboye, una forma estabilizadora, dominante es el medio para establecer un control de una organización que se construye por un proceso similar a una sustracción. En el caso del Hotel Particuliere, el perímetro irregular del lote es neutralizado por Le Pautre con un patio de forma regular, un vacío a cuya forma dominante ordena los espacios de las habitaciones principales. A su vez, la clara voluntad formal y proporcional del espacio de estas habitaciones, que son a veces rectangulares con extremos redondeados, obliga a la utilización de habitaciones aún menores (vacíos) tanto como a la regulación del espesor de los muros (sólidos) para darles forma y acomodarlas entre sí. Se trata de una relación entre formas subordinantes y subordinadas que tiene por objeto controlar la forma y proporción de las primeras a costa de las segundas. En el caso de la Ville Saboye, Le Corbusier decide un perímetro fuerte, regular, al cual subordinar toda la organización de la planta alta. La rampa, escaleras y las habitaciones principales consumen la superficie interior de este perímetro produciendo un sobrante irregular, el patio. Solo los aleros de la terraza devuelven una apariencia de regularidad al patio-terraza del primer piso. En una suerte de teorema Rowe sugiere una fórmula de equivalencias, diciendo que las Galerías Uffizi son a la Unité d'Habitation lo que el patio del Hotel de Beauvais es a la planta alta de la Ville Saboye. Sugiere que el vacío del patio del Hotel, es el opuesto al perímetro de la planta alta de la Ville Saboye.

\section{CASOS CLAVE}

Casi veinte años después de las observaciones de Rowe, el panorama contemporáneo comienza a mostrar arquitectos de vanguardia que hacen un uso explícito de estos recursos proyectuales. En el proyecto para el concurso de la Biblioteca de Francia de OMA, los dibujos describen en negro el espacio genérico, subordinado, y en blanco los espacios principales, formalmente Le Corbusier, Villa Savoye en Poissy

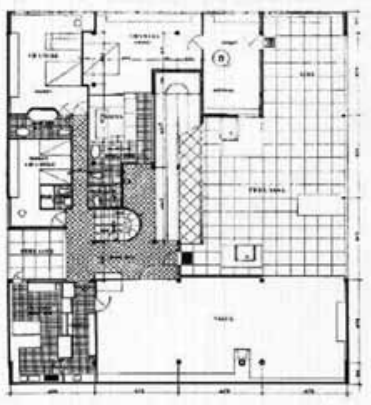

Villa Savoye, plano
Hotel de Beauvais, al zado

F3: Reproducción de Collage City mostrando las plantas altas del Hotel de Beauvais y la Ville Saboye subordinantes en la composición. La forma cuadrada del perímetro repite la operación de 
la Ville Saboye, pero las formas sustraídas son ovales o rectángulos diagonales al perímetro, haciendo que el espacio resultante sea irregular. Todo el argumento formal se basa en esta oposición figural que se hace explícita en la representación utilizada?

En la Ciudad de la Cultura que proyecta Peter Eisenman en 1999 en Santiago de Compostela, Galicia, se advierte un énfasis similar en un poché que se percibe especialmente en las secciones, donde es el mediador entre unas formas exteriores e interiores a las que el arquitecto impone una voluntad formal que espera una total subordinación de los recursos constructivos.

\section{ASTOR PLACE HOTEL, NEW YORK, 1999, HERZOG \& DE MEURON Y OMA}

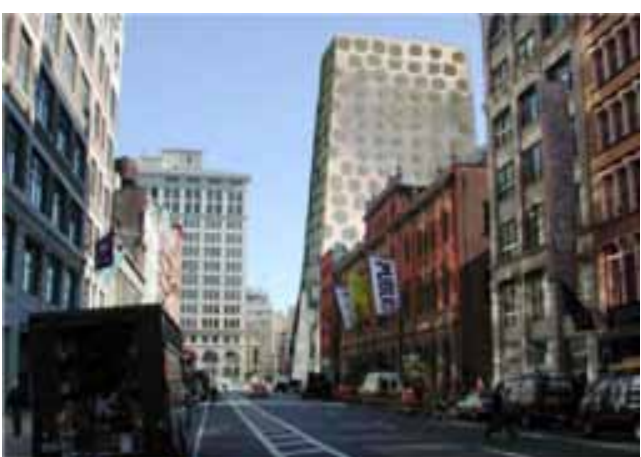

F4: Hotel Astor

hace explícito en la particular forma en que se representa la planta, empleando el negro para los espacios subordinados, al igual que los muros; y el blanco, para el espacio positivo de las habitaciones. Los baños y roperos se asimilan a los muros para producir una forma regular y determinada en las habitaciones. El dibujo asume asi el papel de un discurso, de una explicación un hotel en New York (nunca construido) concebido como un monolito exento que se separa de los edificios veci$n \mathrm{~s}^{8}$. La sugerencia monolítica del volumen exterior se conseguiría por la homogeneidad de sus superficies y por el tratamiento escultural del volumen decreciente, suerte de obelisco truncado, horadado por huecos irregulares, todos diferentes.

Al interior las habitaciones deben acomodarse a un perímetro siempre variable mediante un procedimiento proyectual que se

OMA proyectan
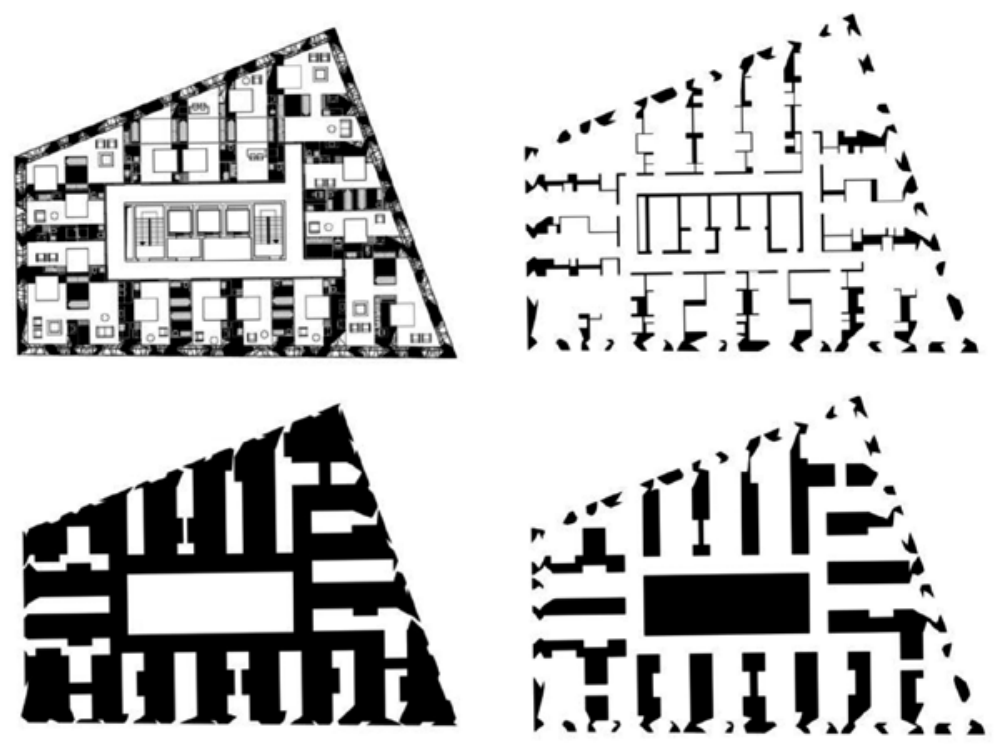

F5: Hotel Astor, planta de los autores / planta de muros / planta espacios / negativo espacios (Dibujos: Francisco Apa y Pia Casals) 
sobre el proceso de proyecto tanto como de la forma correcta de interpretación de lo proyectado. Los dibujos analíticos muestran el contraste entre la representación de los autores y la sección horizontal de los muros sólidos:

\section{MANUEL Y FRANCISCO AIRES MATEUS}

El trabajo de los hermanos Aires Mateus muestra, en una sucesión de proyectos realizados desde el año 1999, la utilización explícita de espacios subordinantes y subordinados, revelando un nuevo interés por la interioridad del espacio ${ }^{9}$. En las casas de Alvalade (1999) y Alentejo (2000-2003) aparece una dialéctica entre llenos y vacíos, y su explícita representación en truirse, tiene un lugar relevante en la monografía que edita Gustavo Gili en $2002^{10}$. La planta está constituida por un perímetro cuadrado con una serie de espacios rectangulares interiores separados por pasillos de ancho constante. En sus propios dibujos, los autores muestran estos corredores en negro, asimilándolos a la representación convencional de un muro.

En la Casa de Alentejo, un perímetro cuadrado con solo dos aberturas define la geometría de la casa. El dibujo de los autores muestra las habitaciones y patios en negro, en contraste con la sala y comedor en blanco. Aparece así enunciada la subordinación formal de los primeros a favor de los segundos. En el sector negro del dibujo, las líneas que delimitan los muros se han invertido, representándose en blanco.

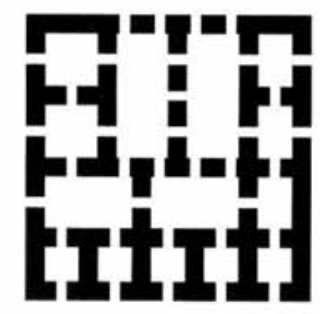
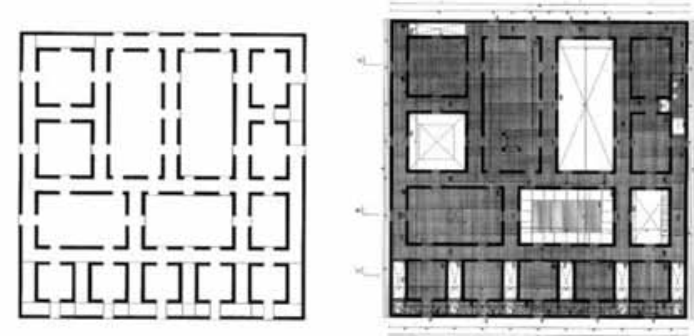
Esta representación hace explícito el principio de organización de la composición. Pero esta subordinación de espacios es también acomodación, no hay sufrimiento en la resolución de la forma de cocina, baños y dormitorios.

F6: Aires Mateus, Casa en Alvalde. Dibujo de los autores

planos de fondo y figura, donde algunas partes vacías son presentadas como sólidos. Aunque la casa en Alvalde (1999) permanece sin cons-
Este modo de concebir y representar el espacio arquitectónico se afirma como una constante en los sucesivos proyectos de las casas en Sesimbra y Alcácer do Sal; y quizá de un modo más espectacular y explícito en la casa en Brejos de Azeitão.
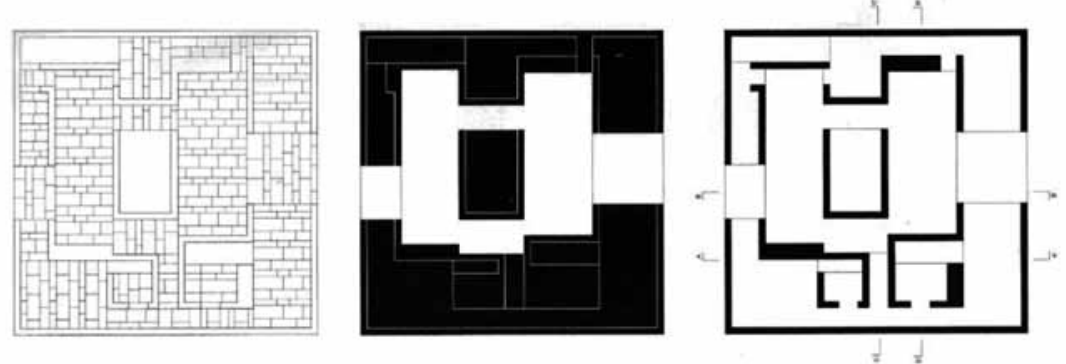

F7: Aires Mateus, Casa en Alentejo. Dibujo de los autores 

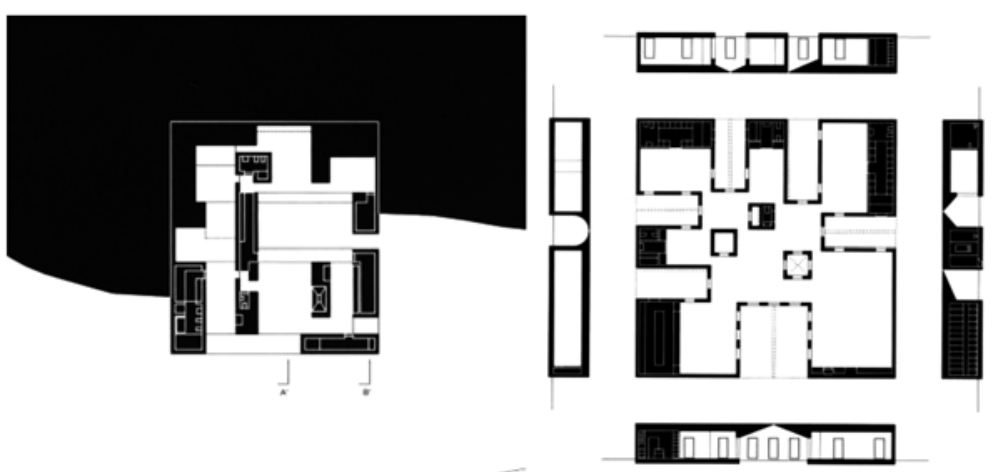

F8: Casa en Sesimbra, planta

F9: Casa en Alcácer do Sal, planta

\section{CASA EN BREJOS DE AZEITÁO, AIRES}

\section{MATEUS, 2000-2003}

Este proyecto consiste en la recuperación de un antiguo almacén de vinos, una construcción de gruesos muros y planta rectangular y un techo a dos aguas que cubre una generosa altura interior. Su transformación en una casa consigue conservar la unidad espacial de esta única nave a pesar de la introducción de un programa que naturalmente supone una serie de pequeñas habitaciones (dormitorios, baños, cocinas). Todas las áreas auxiliares de planta baja se alojan entre las paredes existentes en el perímetro y las nuevas, que las duplican al interior. En la planta alta, una serie de volúmenes flotan en la espacialidad de la nave, son los dormitorios y sus baños. Esta disposición permite ver siempre el gran techo, y tener una percepción constante y unitaria del espacio interior. El esfuerzo estructural para hacerlo posible, permanece oculto bajo la delicadeza de los volúmenes blancos.
La gráfica utilizada por Aires Mateus en el monográfico realizado por $2 \mathrm{G}$, presenta intencionadamente en negro, no sólo los muros, sino también los espacios auxiliares de planta baja (baños, cocinas, bajo-escalera) asimilados a un poché que reconstruye regularmente (aunque de apenas menores dimensiones) el gran espacio unitario de la bodega. Esta intencionalidad revela el procedimiento proyectual, y la esperada percepción espacial.

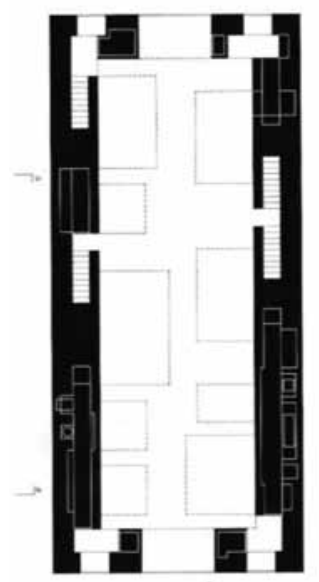

F10: Casa Brejos de Azeitão, Planta baja, dibujo de los autores 

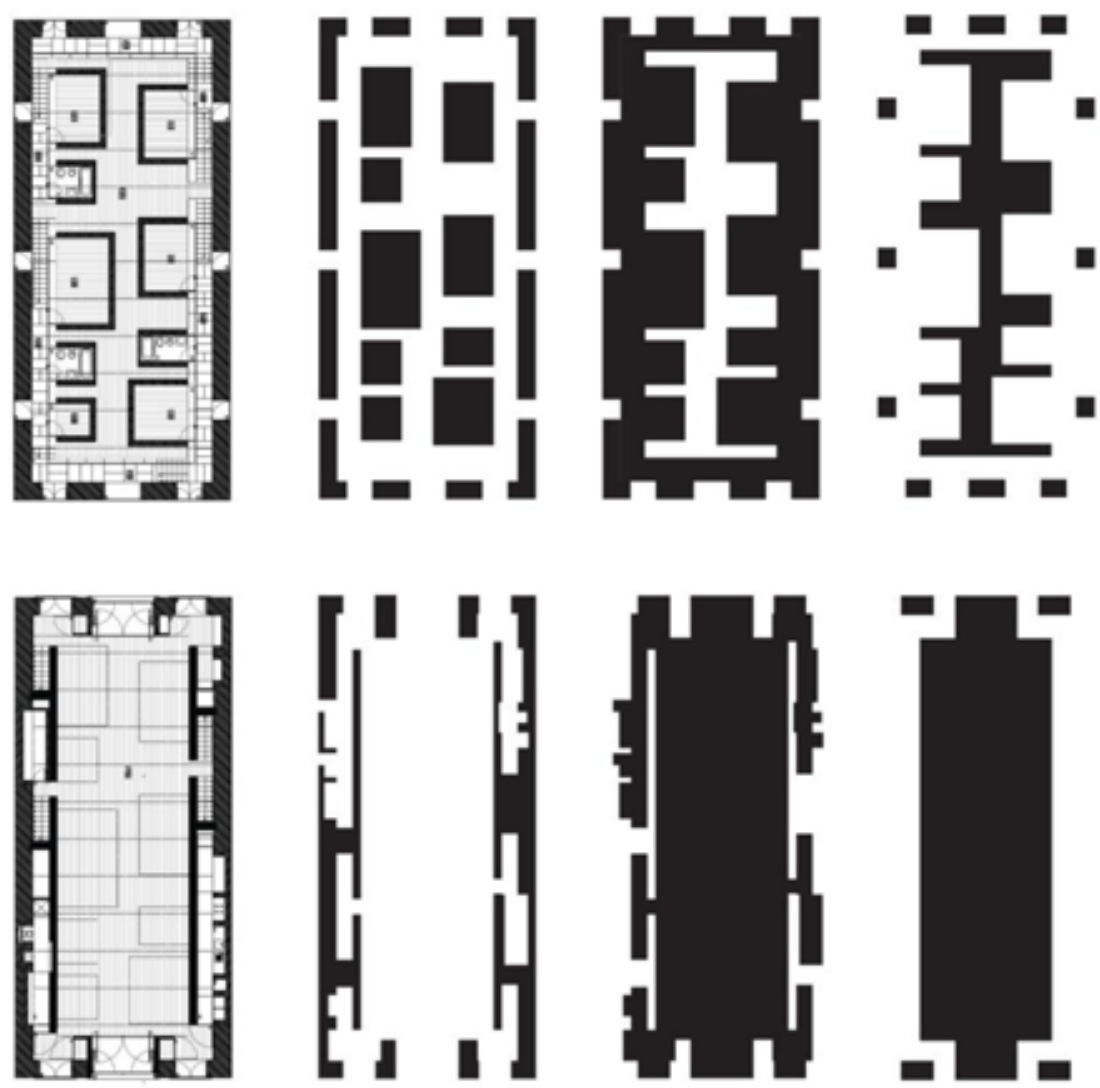

F11: Casa Brejos de Azeitão, planta baja / planta alta: dibujo convencional (izquierda) / muros / negativo muros / espacio percibido (Dibujos: Francisco Apa y Pia Casals)

\section{CAIXA FORUM, HERZOG, DE MEURON Y}

\section{GUGGER, MADRID, 2006}

El nuevo programa de promoción y exhibición artística fue alojado dentro de los gruesos muros de ladrillo de una vieja usina ${ }^{11}$. Al mismo tiempo, debía conservarse la memoria de la usina como parte del patrimonio construido de la ciudad, y proveer un espacio contemporáneo de gran visibilidad y capacidad de atracción.
El proyecto vacía el interior de la nave, pero también demuele la planta baja, convirtiéndola en un espacio continuo con la plaza adyacente que crean los arquitectos. Para ello se realiza un gran esfuerzo estructural que mantiene suspendida la caja de ladrillo. Esto permite una inserción urbana en la que el edificio aparece como un objeto prominente, asimilado 

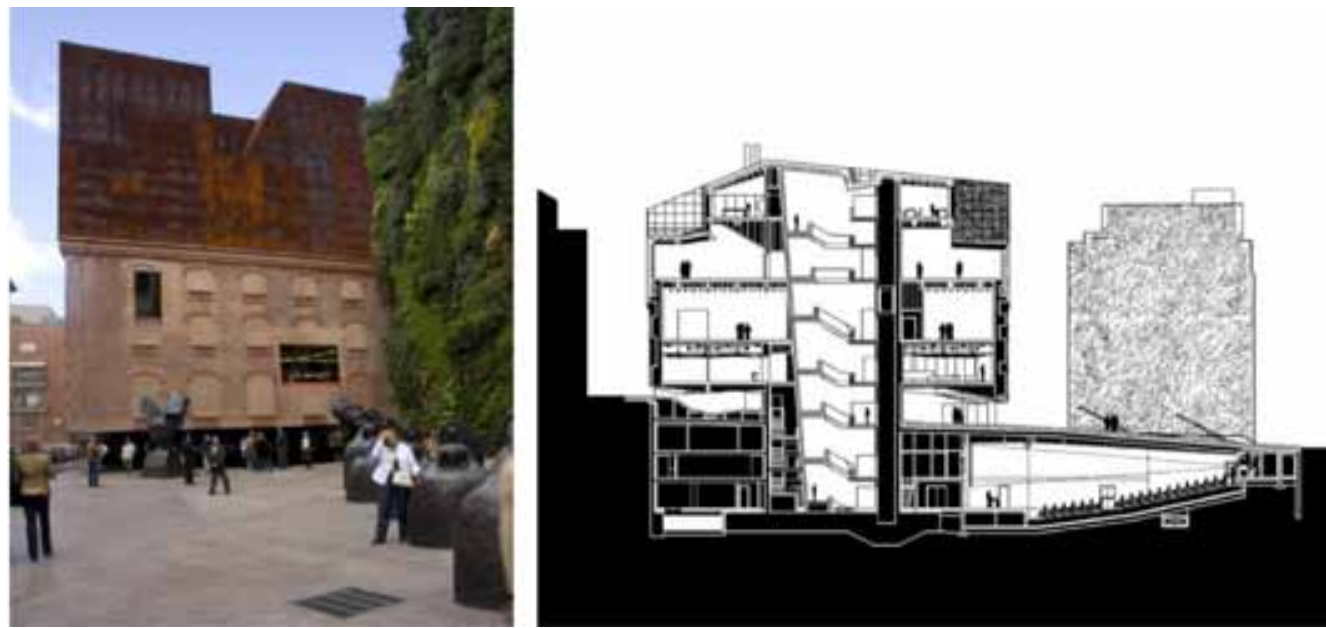

F12: Caixa Forum (Foto: Luis 0'Grady)

F13: Caiza Forum, sección, dibujo de los autores

al tejido urbano continuo y, al mismo tiempo, presidiendo la pequeña plaza. Debido a su planta baja libre, el conjunto edificio-plaza ofrece un contraste con el continuo construido de la edificación tradicional. Es allí donde la noción de sólido-vacío se hace potente. Para anticipar su efecto, los arquitectos utilizan una gráfica en donde el negro representa la edificación de los edificios circundantes tanto como las partes no accesibles de la planta baja del propio edificio.
En el resto de las plantas se utiliza la misma gráfica, discriminando ente los espacios principales, a los cuales se subordinan los espacios secundarios o utilitarios, que son representados en negro, al igual que los muros. Notoriamente en el subsuelo, donde solo se muestra en blanco el auditorio, su foyer y las salas de conferencias, o sea los espacios de acceso al público. Idéntico criterio se sigue en la gráfica de la sección.
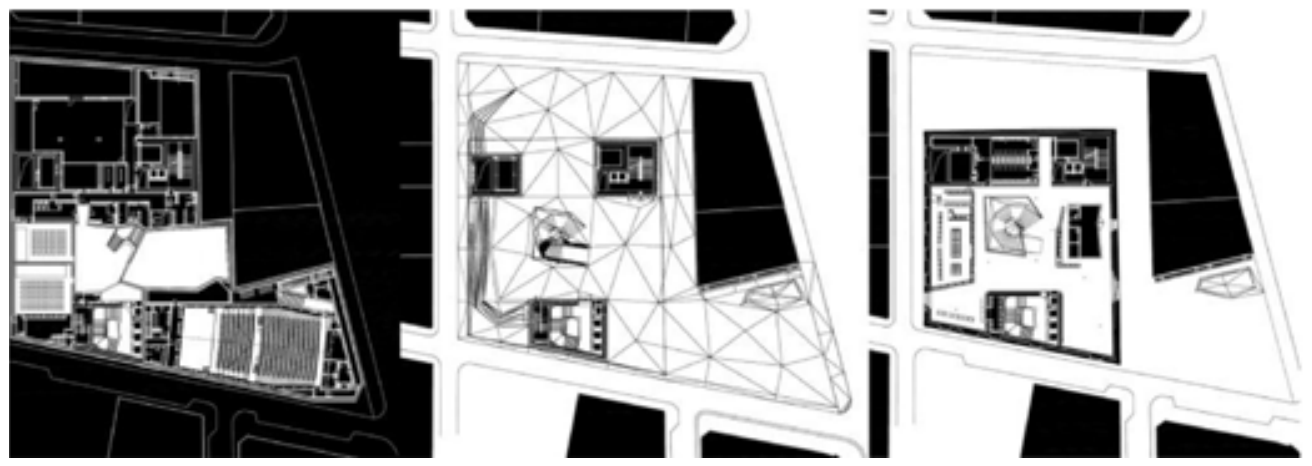

F14: Caixa Forum, subsuelo, planta baja y primer piso (dibujo de los autores) 


\section{CASA WOLF, MAURICIO PEZO Y SOFÍA VON ELLRICHSHAUSEN, SAN PEDRO CHILE, 2006-7}
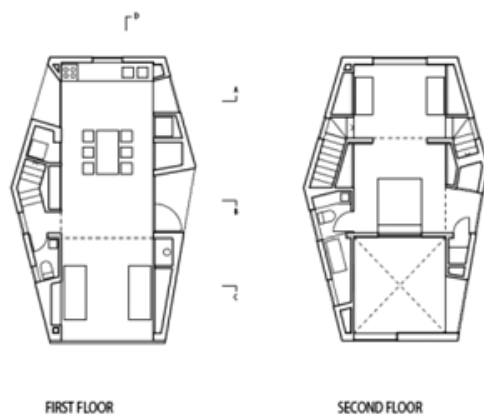

(FFL + +0.00$)$

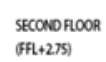

nados, conservando el blanco para el vacío y el negro para el sólido de los espacios subordinantes. Estos dibujos se pueden contrastar con la estricta sección horizontal de los muros sólidos. A su vez, el análisis

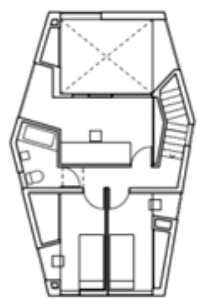

THRO FOOR (FFL +5.50)

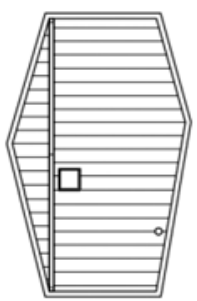

ROOF PLAN (RL+8.50) permite discriminar entre los espacios continuos y discontinuos, independientemente de sus condiciones formales.

F15: Casa Wolf, dibujo original de los autores

Esta pequeña casa de sólo 136 metros cuadrados está construida totalmente en metal, estructura de acero PN24 y cerramiento de aluminio $^{12}$. El programa se divide en tres niveles, ubicándose en la planta baja un largo ambiente rectangular que contiene estar, comedor y cocina. En la siguiente planta se ubica el dormitorio principal asociado a un pequeño estudio, y en el último, los dos dormitorios de los niños. Continuidades espaciales verticales entre los tres niveles, administran la altura de los ambientes y dan una sensación de amplitud a las escasas superficies. Escaleras, baños y roperos, median entre la forma rectangular y regular de estos ambientes principales, y la forma exterior en forma de hexágono deformado.

Aunque los dibujos con que los autores presentan el proyecto son convencionales, nuestros dibujos presentan las plantas con una grafica análoga a la utilizada por Aires Mateus, OMA y Herzog de Meuron. Invirtiendo negro y blanco para representar los espacios subordi-

\section{[ver figura 16, página siguiente]}

La figura del rectángulo central de las habitaciones principales puede corresponderse con el patio del Hotel de Beauvais, o el perímetro del hexágono deformado como el perímetro de la planta alta de la Ville Saboye. En la casa Wolf, la regularidad del corazón y la forma exterior se consiguen gracias a la paciente maniobra de acomodación de todos los elementos menores en el poché intermedio.

\section{ADICIÓN Y SUSTRACCIÓN ICONOGRÁFICA.}

En las obras analizadas, los procedimientos sustractivos y las formas subordinantes y subordinadas resultantes son un medio de organizar las partes del proyecto. De establecer un orden legible entre espacios figuralmente reconocibles. Se trata de un procedimiento de proyecto, de un recurso operacional que per- 

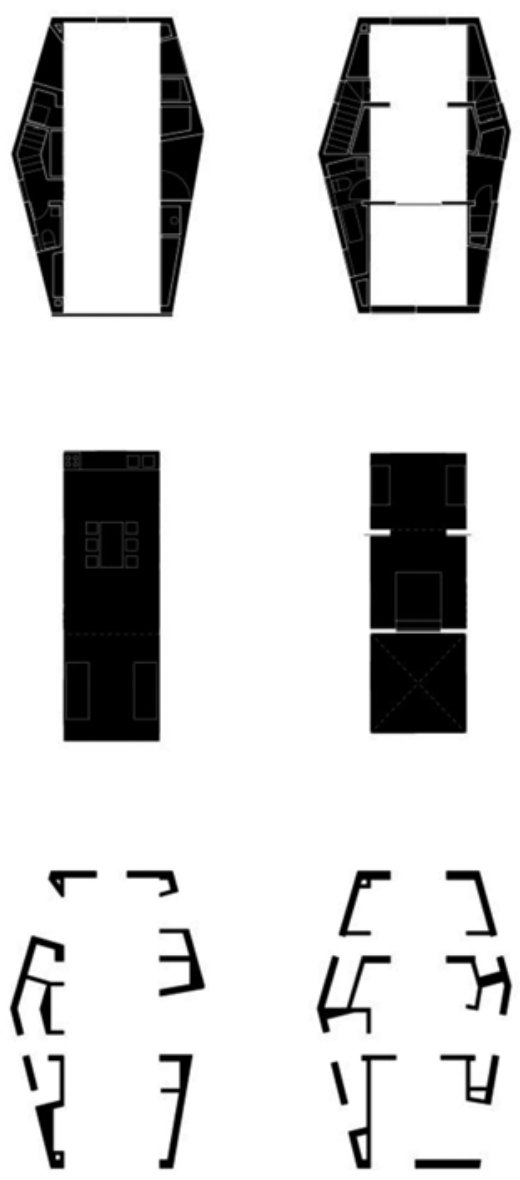

F16: Casa Wolf, de arriba hacia abajo: espacios subordinados / espacios subordinantes / muros (Dibujos: Francisco Apa y Pia Casals)

mite una determinada organización que, a la vez, establece un patrón reconocible, aunque complejo, en la organización de los distintos espacios de uso. Es necesario, sin embargo, establecer una diferencia con otro tipo de proyec-
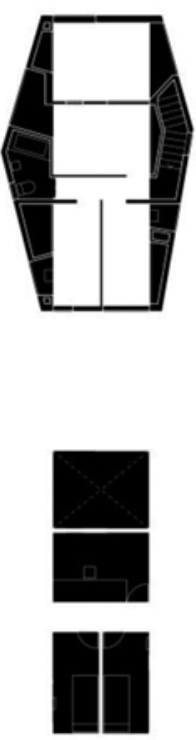

tos contemporáneos donde los procedimientos sustractivos o aditivos son más evidentes, pero radicalmente distintos.

La adición puede ser un proceso de construcción literal cuando se agrega una habitación a una construcción existente, o conceptual, cuando el proyecto se expresa como una suma de partes, aunque no lo sea. Como es notorio, ese exhibicionismo de la adición o la sustracción en general no simplifica, sino que hace aún más complejas las resoluciones constructivas ${ }^{13}$. En el caso del New Museum, diseñado por Kazuyo Sejima y Ryue Nishizawa en la ciudad de New York ${ }^{\bigotimes}$, el edificio se presenta visualmente como el apilamiento de cajas prismáticas. Conseguir esta apariencia obliga a trasladar las cargas siempre a un plano diferente. Pero el esfuerzo estructural del apeo debe permanecer escondido para evitar que interfiera con la metáfora volumétrica de cajas que visualmente deben aparecer como apiladas.

\section{[ver figura 17, página siguiente]}

En la casa View, realizada en Rosario (Argentina) por Johnston-Marklee con Diego Arraigada ${ }^{15}$, 


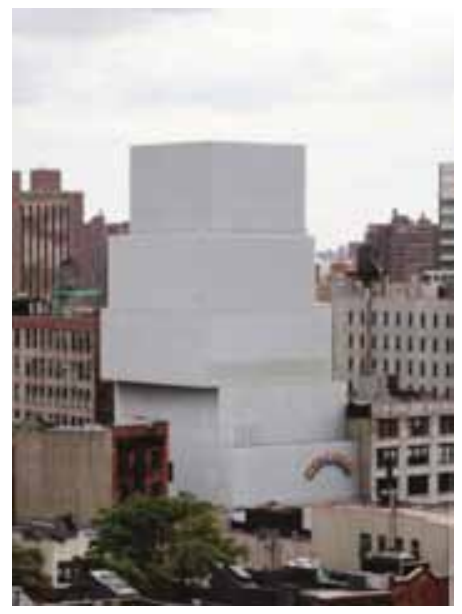

F17: New Museum, New York

la sugestión más potente es la de una sustracción literal de formas esféricas, realizadas sobre una hipotética forma previa, prismática de un lado, circular, del otro. El efecto retórico no podía ser más evidente desde el exterior.

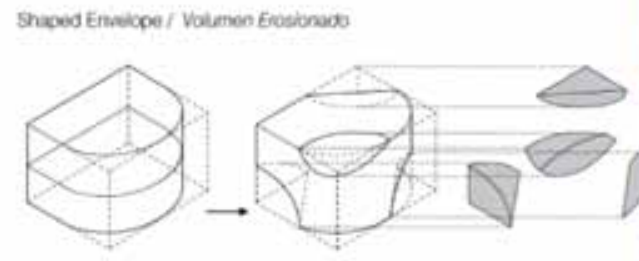

Lo que obliga a que la superficie de hormigón que separa el afuera y el adentro sea una superficie de espesor regular. Esta cuestión hace aún más complejo el cálculo y las armaduras de la cáscara, de pronto esférica, de pronto prismática. Paradójicamente, la simplicidad formal del resultado obliga a una compleja resolución constructiva para dar sustento a los volúmenes de hormigón.

Sin duda, la adición o la sustracción metafórica consiguen un efecto visual espectacular, pero reduciendo el edificio a una imagen icónica, a la que se someten todos los otros aspectos y demandas implícitos en la complejidad del proyecto contemporáneo. En estos contraejemplos, la adición o sustracción de formas simples es el modo de dar un sentido literal a formas complejas. Se trata de conseguir una complejidad legible, que se opondría a la de las arquitecturas complejas analizadas anteriormente, que postergan la comprensión del conjunto a favor de una interrelación más intensa y compleja entre las partes y entre estas y la totalidad.

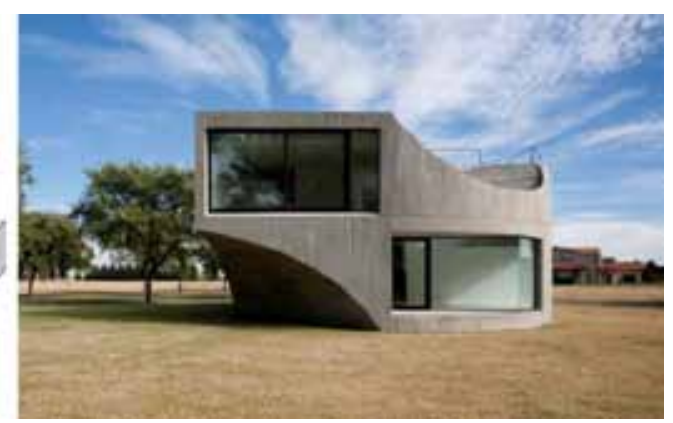

F18: Casa View, diagram de los autores

F19: Casa View, Rosario (Foto: G. Fritegotto)

Hay una correspondencia simétrica entre exterior e interior, es decir que a la concavidad exterior corresponde una convexidad interior.
A diferencia de los casos anteriores, no se trata de un recurso organizacional, sino de un recurso metafórico. Es la simulación de un proceso 
aditivo o sustractivo, como mantenido en suspenso, por la sugestión de su reciente ejecución. Estas diferencias son radicales, porque en los primeros casos se trata de un recurso de proyecto que opera sobre la forma del espacio, en tanto en estos últimos, se trata de un recurso que opera exclusivamente sobre forma objetual del edificio, sobre su iconografía. Se pasa de la composición a la expresión. De la organización del espacio a una retórica visual. Algo que tiene su sentido en la consecución de un éxito comunicacional del edificio, que lo aleja de aquella percepción difícil que adjudicaba Rowe al proyecto moderno ${ }^{16}$ y lo acerca a una lectura fácil, a una elocuencia literal. De algún modo, esta eficiencia metafórica abre la oportunidad

\section{NOTAS}

1 Aunque para la composición clásica, dice Corona Martínez, no todas las partes de la construcción eran Elementos de Arquitectura, así como no cualquier construcción forma parte de la Arquitectura. Corona Martínez, Alfonso, Ensayo sobre el proyecto, CP67, Buenos Aires, 1990.

2 Perls, Fritz, The Gestalt Approach \& Eye Witness to Therapy, Science and Behavior Books, Palo Alto, 1973.

3 Asi lo manifiesta Manuel Aires Mateus, en conversación con Fernando Diez, Lisboa, Abril de 2008.

4 Durand, J.N.L., Précis de Leçons d'architecture, Paris, 1819.

5 Puede verse Corona Martínez, Alfonso, El proyecto, la influencia de los medios analógicos en la arquitectura, Mac Gaul, Buenos Aires, 1976.

6 Rowe, Colin y Koetter, Fred, Ciudad Collage, Gustavo Gili, Barcelona, 1981.

$7 S, M, L, X L$. , OMA, Rem Koolhaas, Bruce Mau, 010 Publishers, Rotterdam, 1995.

8 http://www.oma.eu/index.php.option.com_ projects\&view.portal\&id637\&Itemid10. para una popularización de la arquitectura y posibilitarle un protagonismo en el mundo de imágenes icónicas que dominan el escenario contemporáneo ${ }^{17}$ pero, también, a una perdida de densidad interna, de complejidad y riqueza espacial, obsesionada por el aspecto visual antes que por la cualificación espacial.

Las obras aquí analizadas sugieren, por el contrario, que algunos proyectos contemporáneos vuelven sobre el espacio como centro del proyecto, retomando una tradición compositiva donde la sustracción puede ser una herramienta de cualificación y organización formal, estableciendo la posibilidad de una jerarquía más compleja y de espacios también más diversificados.

9 Manuel Aires Mateus confirma esta percepción a diferencia de la mayoría de los arquitectos contemporáneos, nos interesa el espacio. (en conversación con Fernando Diez, Lisboa, 2008).

10 2G N.28, Aires Mateus, João Belo Rodeia, Alberto Campo Baeza (textos), Barcelona, 2002.

11 Summa+100, Buenos Aires, Abril 2009, pp.42-47.

12 Summa+98, Buenos Aires, diciembre 2008, pp.128-131.

13 Repitiendo la observación de Evans sobre el deconstructivismo en Evans, Robin, The projective cast, The MIT Press, London, 1995.

14 Summa+104, Buenos Aires, Noviembre 2009, pp.58-65. 15 Summa+104, Buenos Aires, Noviembre 2009, pp.38-43. 16 Rowe, Colin, Manierismo, Arquitectura Moderna y otros ensayos, GG Reprints, Gustavo Gili, Barcelona, 1999, p.5.

17 Puede verse Josep Lluis Mateo, Iconoclastia: News from a post-iconic World. Architectural Papers IV: Iconoclastia, Chair of Prof. Dr. Josep Lluís Mateo, ETH-Zurich. Switzerland , 2009. 


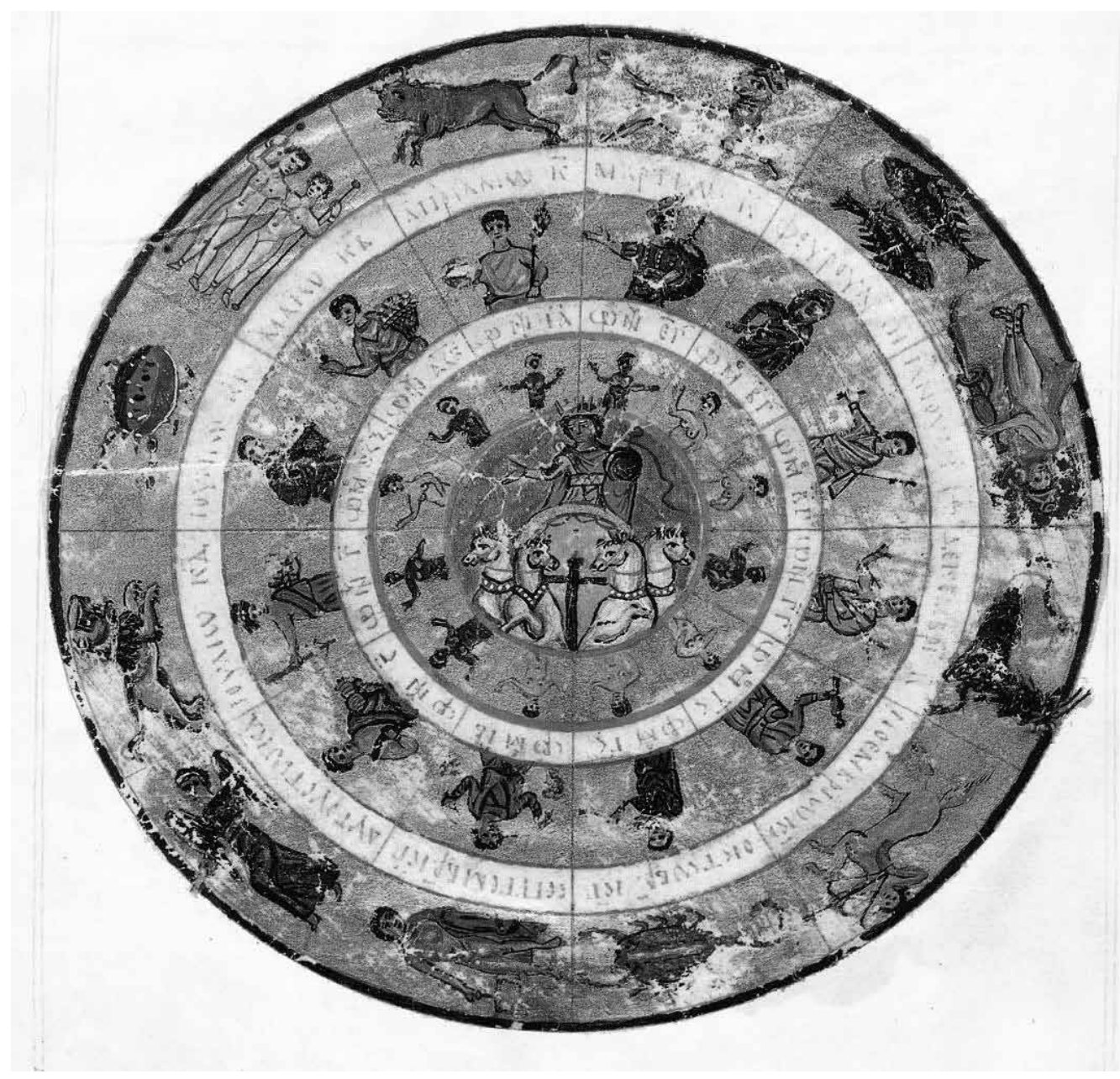

